Results We observe that expression levels of components of the notch signalling pathway vary strikingly between LM-, PM- and NE-derived populations during differentiation, with a distinct peak in Notch3 expression observed early in the development of NE-derived populations. Notch3 knockdown, through either siRNA or shRNA approaches, inhibits the differentiation of NE-derived SMCs as observed by markedly impaired expression of SMC-specific markers, whilst LM- and PM-derived population show an additional lineage specific phenotype.

Conclusions/Implications These results provide further insights into the pathology observed in CADASIL patients, with NE-derived populations showing Notch3 dependence for differentiation, and maturation. We believe that this in vitro lineage-specific Notch3 knockdown phenotype may translate to the pathophysiology observed during disease. To further elucidate this, we aim to unravel the mechanisms through which Notch3 signalling regulates these lineages, utilising both next generation sequencing to identify novel pathways through which Notch3 may regulate SMC biology, and through the generation of induced pluripotent stem cells (iPSC) from CADASIL patients. These approaches may lead to the identification of novel therapeutic targets in the management of CADASIL.

191

\section{NOTCH3 REGULATES HUMAN VASCULAR SMOOTH MUSCLE CELL DEVELOPMENT AND FUNCTIONALITY IN A LINEAGE SPECIFIC MANNER: IMPLICATIONS IN THE PATHOLOGY OF CADASIL}

W Bernard, A Granata, S Sinha The Univeristy of Cambridge

doi:10.1136/heartjnl-2013-304019.191

Introduction CADASIL (Cerebral Autosomal Dominant Arteriopathy with Subcortical Infarcts and Leukoencephalopathy) is characterised by degeneration of the smooth muscle cells (SMC) within the cerebrovasculature, resulting in recurrent subcortical ischemic episodes and vascular dementia. Mutations of the singlepass transmembrane protein Notch3 have been described as the causative component of CADASIL. Whilst Notch3 is expressed in all vascular SMCs, the major pathology observed in CADASIL is primarily located within the cerebrovasculature, with other vascular beds remaining predominantly unaffected. SMCs have diverse embryological origins, and we hypothesise that these origins may regulate the physiology and regional disease pre-disposition within this cell type.

Methods To address this anomaly, we have utilised our recently developed protocol which allows the generation of SMCs, from human embryonic stem cells (hESC), through multiple developmental lineage intermediates: lateral-plate (LM) and paraxial mesoderm (PM), and neuroectoderm (NE). By integrating either siRNA mediated knockdown of Notch3 at critical time points, or the generation of stably expressing Notch3 shRNA hESC lines, we are beginning to address the role of Notch3 in human SMC development and pathology. 